\title{
Catheter-Related Leuconostoc Bacteraemia in a Pregnant HIV-Infected Woman
}

\author{
Darren W Wong ${ }^{\mathrm{a}}$, William Yang ${ }^{\mathrm{a}}$, Ole Vielemeyer ${ }^{\mathrm{b}, \mathrm{c}}$
}

\begin{abstract}
Leuconostoc species are Gram-positive coccobacilli that are members of the family of streptococcaceae. They are rarely encountered in clinical medicine and in the past were considered culture contaminants. More recently they have been recognized as a potential pathogen causing opportunistic infections. Unlike other Gram-positive cocci Leuconostoc spp. are intrinsically resistant to vancomycin. Here we present a case of fever and Leuconostoc spp. bacteraemia in a pregnant HIV-infected woman requiring parenteral nutrition via central venous access for severe hyperemesis gravidarum. The infection was successfully treated with catheter removal. To our knowledge this is the first report of bacteremia with this organism in pregnancy as well as in an adult HIV infected patient. Issues of possible misidentification of this organism as alphahemolytic streptococcus are discussed.
\end{abstract}

Keywords: Leuconostoc; Bacteremia; Immunosuppression; Pregnancy; Vancomycin resistance; Total parenteral nutrition; TPN

\section{Introduction}

One of the most feared complications of central-venous catheter use is line-related bacteremia. In the majority of cases the causative organism are staphylococci originating from the patient's skin. Thus, intravenous vancomycin is frequently used as empiric therapy until results of blood

\footnotetext{
Manuscript accepted for publication July 27, 2012

${ }^{a}$ Department of Internal Medicine, Drexel University College of Medicine, Philadelphia, PA, USA

${ }^{\mathrm{b}}$ Department of Internal Medicine, Division of Infectious Diseases and HIV Medicine, Drexel University College of Medicine, Philadelphia, Pennsylvania, USA

${ }^{\mathrm{c} C}$ Corresponding author: Ole Vielemeyer, 245 N. 15th Street,

Philadelphia, Pennsylvania 19102, USA.

Email: ole.vielemeyer@drexelmed.edu
}

doi: http://dx.doi.org/10.4021/jmc822w cultures are known. Infrequently, vancomycin-resistant Gram-positive organisms are encountered which are almost always later identified as vancomycin-resistant enterococci (VRE). Identification of a non-enterococcal vancomycinresistant Gram-positive organism, in a clinical microbiology laboratory fortunately remains a rare event. If the Gram stain suggests staphylococcal species, vancomycin-resistant $S$. aureus (VRSA) must be considered. If the bacteria belong to the streptococcus (but not enterococcus) family Leuconostoc spp. which are intrinsically resistant to vancomycin, are most likely encountered. While Leuconostoc spp. has been used in the food industry for many years, their importance as a pathogen in human infection has only recently been established. Here we describe what we believe to be the first reported case of Leuconostoc bacteremia in a patient whose immune system was compromised by HIV-infection and pregnancy.

\section{Case Report}

A 24-year-old pregnant African American woman in her 13th week of gestation presented to our emergency department with a one-day history of fevers, chills, and night sweats. Throughout her pregnancy she had been battling severe hyperemesis gravidarum requiring two prior hospital admissions for intractable nausea and vomiting. A peripherally inserted central venous catheter (PICC) for administration of total parenteral nutrition (TPN) and intravenous antiemetics had been placed 18 days prior to the onset of fever. The patient denied cough, chest pain or dysuria. Her gastrointestinal symptoms related to the hyperemesis gravidarum had remained unchanged. The patient's medical history was otherwise significant for chronic HIV-infection with a relatively preserved $\mathrm{CD}^{+} \mathrm{T}$-lymphocyte count $(358$ cells $/ \mu \mathrm{L}$ ). There was no intravenous drug use history.

On admission the patient was febrile to $38.9^{\circ} \mathrm{C}(102.0$ $\left.{ }^{\circ} \mathrm{F}\right)$, tachycardic but appeared non-toxic. Aside from mild abdominal tenderness physical examination was unremarkable with no findings suggestive of a focal source of infection. The PICC site had no erythema, induration, tenderness, or drainage. Laboratory studies showed a WBC count of 5.9 
cells/ $\mu \mathrm{L}$ with a left shift. Urinalysis and urine culture as well as chest radiography were normal.

Two sets of blood cultures were obtained via peripheral veins and empiric treatment with intravenous vancomycin was begun. Within 24 hours both sets turned positive. Gramstains from all four bottles revealed Gram-positive cocci in pairs and chains. The isolates grew readily on blood agar plates and were reported the following day as "presumptive $\alpha$-hemolytic streptococci not enterococci". The PICC line was removed; vancomycin was continued. The patient subsequently defervesced. Repeat blood cultures drawn on hospital days 2 and 5 remained sterile. The blood isolate was eventually identified as Leuconostoc spp., resistant to vancomycin, susceptible to penicillin on hospital day five. In addition, growth of Candida parapsilosis (only seen on sub-cultured plates used to work up the Gram positive cocci) was reported on day six. The patient remained afebrile and clinically stable off effective antimicrobial therapy. She was discharged in improved condition without further antibiotics. No relapse of bacteremia/fungemia occurred throughout the remainder of her pregnancy and she delivered a healthy HIVnegative baby boy six months later.

\section{Discussion}

Leuconostoc spp. is Gram-positive coccobacilli, which form pairs or chains and belong to the family of streptococcaceae. They grow as $\alpha$ - or non-haemolytic gray-white or colourless colonies on blood agar. The organism is found in plant matter, fermenting vegetables, dairy products, wines, and meats. Owing to the paucity of documented cases the exact mode of transmission to humans and the pathogenesis of leuconostoc infections remain poorly defined. Furthermore, misidentification and misreporting of Leuconostoc spp. has been a problem. This is in part because of the infrequent occurrence and in part because the organism shares many morphological and biochemical characteristics with viridians streptococci. In one case series, four of six clinical isolates were initially identified as S. sanguis by automated microbial identification systems [1]. Misidentification as Streptoccocus salivaris, Streptococcus pneumonia, and Streptococcus avium has also been reported.

Possible predisposing factors for Leuconostoc bacteraemia include immunosuppression, prolonged hospitalization, presence of a central venous catheter, need for TPN, disruption of bowel mucosa and long-term antibiotic treatment, especially with vancomycin. Cases after liver and bone marrow transplantation, in a patient with end-stage liver disease, in a diabetic with multiple abscesses, in a severe burn victim, and in the paediatric population associated with AIDS or short gut syndrome have all been described [1-18]. Invasive infections have also been reported in patients with no known risk factors [19].
Unlike most Gram-positive bacteria, Leuconostoc spp. is intrinsically resistant to vancomycin. The organism is able to synthesize an altered vancomycin target at the terminus of the peptidoglycan cell wall leading to a manifold log reduction in affinity [20]. Successful treatment strategies include removal of indwelling catheters and administration of intravenous penicillin [1]. Alternative antimicrobials that have been suggested include clindamycin, ampicillin, macrolides, minocycline, aminoglycosides, and more recently daptomycin and tigecycline $[9,17]$.

In our case, use of TPN, presence of an indwelling catheter and maybe an abnormal gut mucosa due to repeated bouts of vomiting were the likely risk factors for the patient's bacteremia with this unusual organism. Likely contributing was also her immunosuppressed state related to her chronic HIV-infection and current pregnancy. We believe that our patient was adequately and appropriately treated with catheter removal and discontinuation of parenteral nutrition alone, a strategy that has been described before $[1,2]$.

Bacteraemia due to Leuconostoc spp. remains an uncommon clinical entity. To our knowledge, this is the first case in an adult HIV-infected patient as well as the first case seen during pregnancy. When cultures yield non-enterococcal Gram-positive cocci in pairs and chains with a decreased susceptibility to vancomycin, infection due to Leuconostoc spp. should always be suspected. In our case, clinicians were informed of the presence of a vancomycin-resistant organism in the blood only after five days. Since vancomycin remains a commonly used choice of empiric therapy for bacteremia with Gram-positive organisms efforts should be made to alert clinicians of the possibility of vancomycin-resistant Leuconostoc spp. as soon as possible, thus prevent any delay in initiation of appropriate antibiotic therapy.

\section{Grant Support}

There was no grant supporting the preparation of this manuscript.

\section{Financial Disclosures and Conflict of Interest}

All authors declare that there is no conflict of interest and there are no financial disclosures related to this case.

\section{References}

1. Handwerger S, Horowitz H, Coburn K, Kolokathis A, Wormser GP. Infection due to Leuconostoc species: six cases and review. Rev Infect Dis. 1990;12(4):602-610.

2. Bernaldo de Quiros JC, Munoz P, Cercenado E, Hernandez Sampelayo T, Moreno S, Bouza E. Leuconos- 
toc species as a cause of bacteremia: two case reports and a literature review. Eur J Clin Microbiol Infect Dis. 1991;10(6):505-509.

3. Peters VB, Bottone EJ, Barzilai A, Hyatt AC, Blank S, Hodes DS. Leuconostoc species bacteremia in a child with acquired immunodeficiency syndrome. Clin Pediatr (Phila). 1992;31(11):699-701.

4. Carapetis J, Bishop S, Davis J, Bell B, Hogg G. Leuconostoc sepsis in association with continuous enteral feeding: two case reports and a review. Pediatr Infect Dis J. 1994;13(9):816-823.

5. Dhodapkar KM, Henry NK. Leuconostoc bacteremia in an infant with short-gut syndrome: case report and literature review. Mayo Clin Proc. 1996;71(12):1171-1174.

6. Espinoza R, Kusne S, Pasculle AW, Wada S, Fung J, Rakela J. Leuconostoc bacteremia after liver transplantation: another cause of vancomycin resistant gram-positive infection. Clin Transplant. 1997;11(4):322-324.

7. Jimenez-Mejias ME, Becerril B, Gomez-Cia T, Del Nozal M, Palomino-Nicas J. Bacteremia caused by Leuconostoc cremoris in a patient with severe burn injuries. Eur J Clin Microbiol Infect Dis. 1997;16(7):533-535.

8. Monsen T, Granlund M, Olofsson K, Olsen B. Leuconostoc spp. septicaemia in a child with short bowel syndrome. Scand J Infect Dis. 1997;29(3):310-311.

9. Golan Y, Poutsiaka DD, Tozzi S, Hadley S, Snydman DR. Daptomycin for line-related Leuconostoc bacteraemia. J Antimicrob Chemother. 2001;47(3):364-365.

10. Templin KS, Crook T, Riley T, 3rd, Whitener C, Aber RC. Spontaneous bacterial peritonitis and bacteremia due to Leuconostoc species in a patient with end-stage liver disease: a case report. J Infect. 2001;43(2):155157.

11. Vagiakou-Voudris E, Mylona-Petropoulou D, Kalogeropoulou E, Chantzis A, Chini S, Tsiodra P, MalamouLada E. Multiple liver abscesses associated with bacteremia due to Leuconostoc lactis. Scand J Infect Dis.
2002;34(10):766-767.

12. Helali A, McAlear D, Osoba A. Leuconostoc bacteremia in a child with short-gut syndrome. Saudi Med J. 2005;26(2):311-313.

13. Florescu D, Hill L, Sudan D, Iwen PC. Leuconostoc bacteremia in pediatric patients with short bowel syndrome: case series and review. Pediatr Infect Dis J. 2008;27(11):1013-1019.

14. Tommasi C, Equitani F, Masala M, Ballardini M, Favaro $\mathrm{M}$, Meledandri $\mathrm{M}$, Fontana $\mathrm{C}$, et al. Diagnostic difficulties of Lactobacillus casei bacteraemia in immunocompetent patients: a case report. J Med Case Rep. 2008;2:315.

15. Yamazaki R, Mori T, Sugita K, Aisa Y, Ikeda Y, Okamoto $S$. Leuconostoc septicemia in a neutropenic patient with acute myelogenous leukemia relapsed after allogeneic peripheral blood stem cell transplantation. Transpl Infect Dis. 2009;11(1):94-95.

16. Ishiyama K, Yamazaki H, Senda Y, Yamauchi H, Nakao $\mathrm{S}$. Leuconostoc bacteremia in three patients with malignancies. J Infect Chemother. 2011;17(3):412-418.

17. Lee MR, Huang YT, Lee PI, Liao CH, Lai CC, Lee LN, Hsueh PR. Healthcare-associated bacteraemia caused by Leuconostoc species at a university hospital in Taiwan between 1995 and 2008. J Hosp Infect. 2011;78(1):4549

18. Shin J, Her M, Moon C, Kim D, Lee S, Jung S. Leuconostoc bacteremia in a patient with amyloidosis secondary to rheumatoid arthritis and tuberculosis arthritis. Mod Rheumatol. 2011;21(6):691-695.

19. Casanova-Roman M, Rios J, Sanchez-Porto A, Gomar JL, Casanova-Bellido M. Leuconostoc bacteremia in a healthy infant. Minerva Pediatr. 2003;55(1):83-86.

20. Neuhaus FC. Role of the omega loop in specificity determination in subsite 2 of the D-alanine:D-alanine (D-lactate) ligase from Leuconostoc mesenteroides: a molecular docking study. J Mol Graph Model. 2011;30:31-37. 